\title{
Probing the excitation spectrum of polariton condensates
}

\author{
Michiel Wouters ${ }^{1}$ and Iacopo Carusotto ${ }^{2,3}$ \\ ${ }^{1}$ Institute of Theoretical Physics, Ecole Polytechnique Fédérale de Lausanne (EPFL), CH-1015 Lausanne, Switzerland \\ ${ }^{2}$ CNR-INFM BEC Center and Dipartimento di Fisica, Università di Trento, I-38050 Povo, Italy \\ ${ }^{3}$ Institute of Quantum Electronics, ETH Zürich, 8093 Zürich, Switzerland \\ (Received 29 September 2008; revised manuscript received 27 November 2008; published 13 March 2009)
}

\begin{abstract}
We propose a four-wave mixing experiment to probe the elementary excitation spectrum of a nonequilibrium Bose-Einstein condensate of exciton-polaritons under nonresonant pumping. Analytical calculations based on mean-field theory show that this method is able to reveal the characteristic negative energy feature of the Bogoliubov dispersion. Numerical simulations including the finite spatial profile of the excitation laser spot and a weak disorder confirm the practical utility of the method for realistic condensates.
\end{abstract}

DOI: 10.1103/PhysRevB.79.125311

PACS number(s): 03.75.Kk, 71.36.+c, 78.47.nj

\section{INTRODUCTION}

A series of remarkable experiments have recently demonstrated the occurrence of Bose-Einstein condensation in systems of exciton-polaritons in semiconductor microcavities. ${ }^{1-5}$ These observations, together with parallel ones on magnon condensation in magnetic solids, ${ }^{6}$ are opening the way to the investigation of the Bose-Einstein condensation phase transition and of Bose-condensed quantum fluids in completely new regimes far from thermal equilibrium where the state of the condensate is no longer determined by a thermal equilibrium condition but by a dynamical balance between driving and dissipation. ${ }^{7}$

A key quantity in the theory of quantum fluids is the dispersion of the elementary excitations, which determines the dynamics of the system in response to external perturbations and, in particular, plays a central role in determining its superfluidity properties. ${ }^{8}$ While detailed knowledge is nowadays available of the phonon and roton branches of liquid helium and of the Bogoliubov modes of dilute atomic gases, ${ }^{9}$ not much experimental work has been performed yet on polariton condensates. On one hand, pioneering luminescence ${ }^{10}$ and pump-and-probe experiments ${ }^{11,12}$ have addressed the excitations of resonantly pumped condensates in an optical parametric oscillator (OPO) configuration; on the other hand, luminescence experiments suggesting a linear dispersion of the elementary excitations in nonresonantly excited polariton condensates have been recently reported. ${ }^{13}$ Still, none of these works has provided complete evidence of the peculiar features that were predicted to appear because of the nonequilibrium nature of polariton condensates. ${ }^{14-16}$

From a different standpoint, while quite some evidence is available for "off-branch" scattering processes in OPO configurations, ${ }^{10,11}$ no observation of the related negative energy "ghost" branch that is expected to appear under nonresonant pumping as a consequence of polariton-polariton interactions has been reported yet. Most likely, this stems from the fact that experiments were based on luminescence spectroscopy ${ }^{13}$ so that the emission from the ghost branch was easily masked by the much stronger background of the condensate emission. A few recent theoretical works have proposed more refined schemes that appear suited to overcome this difficulty and detect this elusive branch by looking either at the absorption ${ }^{17}$ and resonant Rayleigh scattering ${ }^{18}$ spectra or at the density response to an external perturbation. ${ }^{19}$

In the present work, we push these ideas forward and we propose a simple four-wave mixing (FWM) spectroscopy scheme to measure the dispersion of all elementary excitations branches of a nonresonantly pumped polariton condensate: of the three incident beams needed in a FWM experiment, two are provided by the condensate and one by an external laser field. The dispersion of excitations is inferred by scanning the energy and wave vector of this latter beam and by recording the transmitted, reflected, and/or four-wave mixed beams. A first application of related FWM techniques to polariton systems across the parametric oscillation threshold was reported in Ref. 20, but no specific interest was paid to the excitation modes of the condensate itself.

The mean-field Gross-Pitaevskii formalism to describe the polariton condensate dynamics that was introduced in Ref. 16 is briefly reviewed in Sec. I and then applied in Sec. II to describe the FWM response of polariton condensates. Closed formulas are extracted for the transmission, reflection, and FWM signals in the spatially homogeneous case and then used to discuss the main features of the spectra. Generalization to the experimentally relevant case of finite-size condensate is investigated in Sec. III: the efficiency of the FWM method for realistic condensates is confirmed by means of a numerical solution of the polariton Gross-Pitaevskii equation (GPE) in nonuniform geometries. Conclusions are finally drawn in Sec. IV.

\section{ELEMENTARY EXCITATION SPECTRUM}

As a consequence of the short lifetime of polaritons in state-of-the-art microcavities, the polariton condensate can hardly be considered as a thermal equilibrium object: continuous external pumping is necessary to keep the condensate in a stationary state, and the state of the condensate is determined by a dynamical balance between pumping and dissipation. While the momentum distribution of large systems still appears to follow at large energies the typical exponential law of equilibrium statistical mechanics, ${ }^{1-3}$ clear evidence of the nonequilibrium nature of the polariton condensate has been observed in the ballistic outward polariton 


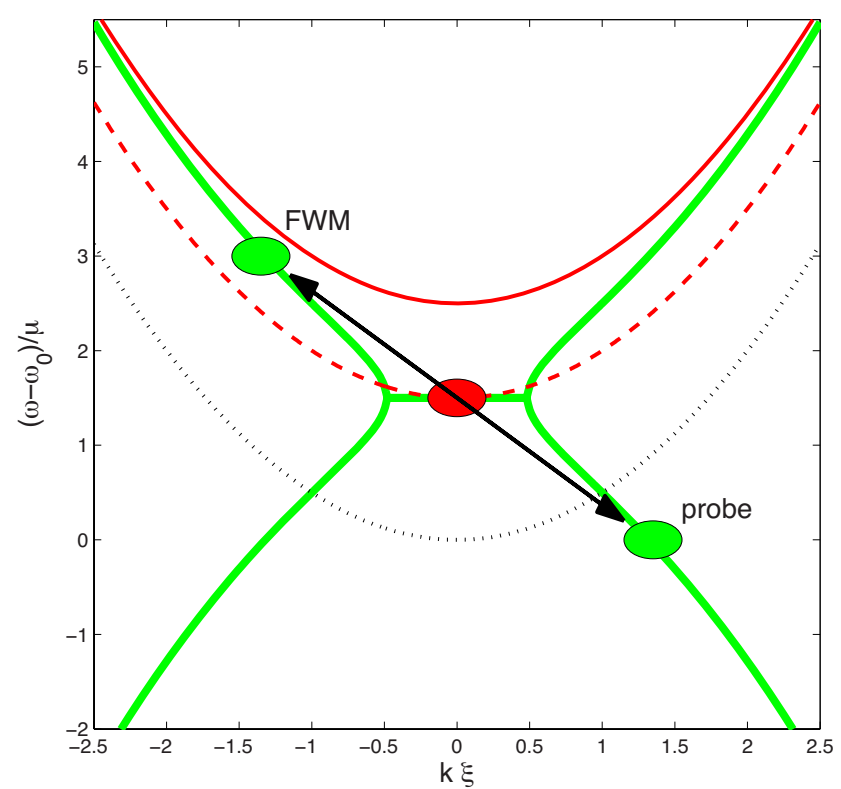

FIG. 1. (Color online) Plot of the elementary excitation spectrum in a spatially homogeneous geometry and sketch of the proposed FWM experiment. The central dot indicates the wave vector $k=0$ and the energy $\omega_{c}$ of the condensate. The heavy solid line is the dispersion [Eq. (1)] of the elementary excitations on top of the condensate. The arrows show the parametric scattering process on which our proposed scheme is based: the condensate is probed at the lower dot, and the response is probed via the emission at the upper dot. The dotted line is the dispersion of free polaritons at linear regime. The solid and dashed lines include the blue shift due to condensate-condensate and condensate-reservoir interactions as mentioned in the text. System parameters: $\gamma=\Gamma=g n_{c}$ and $g_{R} n_{R}$ $=0.5 g n_{c}$.

flow from small-sized condensates ${ }^{21,22}$ as well as in the spontaneous appearance of vortices in the presence of a significant disorder potential. ${ }^{23}$

Several theoretical papers have recently investigated the effect of the nonequilibrium condition on the elementary excitation spectrum ${ }^{14,16}$ and, in particular, have pointed out the diffusive nature of the Goldstone mode at low wave vectors. In what follows, we shall adopt the point of view of our previous paper ${ }^{16}$ where the dynamics of a nonequilibrium condensate was discussed at the mean-field level in terms of a generalized nonequilibrium Gross-Pitaevskii equation.

This gives the following form for the dispersion of the elementary excitations on top of a spatially homogeneous nonequilibrium condensate:

$$
\omega_{ \pm}(k)-\omega_{c}=-i \Gamma / 2 \pm \sqrt{\left[\omega_{\mathrm{Bog}}(k)\right]^{2}-\Gamma^{2} / 4},
$$

which is plotted in Fig. 1 as a heavy solid line. In the low- $k$, diffusive region of the spectrum the \pm signs correspond to, respectively, the phase and the density branches. In the high- $k$ region where the standard equilibrium Bogoliubov dispersion is recovered, they instead correspond to the normal and anomalous ghost branches. In what follows, specific attention will be paid to the ghost branch which is a signature of coherent polariton-polariton interactions. ${ }^{9}$
The condensate energy $\omega_{c}$ (central dot) is blue shifted with respect to the bottom of the lower polariton branch (dotted line) by the condensate-condensate and condensatereservoir interactions, $\omega_{c}=\omega_{0}+g n_{c}+g_{R} n_{R}$. Here, $n_{c}$ and $n_{R}$ are the densities, respectively, of the condensate and of the exciton reservoir, while the $g$ and $g_{R}$ coupling constants characterize the interactions, respectively, between a pair of condensate polaritons and between the condensate polaritons and reservoir excitons. The Bogoliubov spectrum of equilibrium condensates has the standard form $\omega_{\mathrm{Bog}}(k)=\left[\varepsilon_{k}\left(\varepsilon_{k}\right.\right.$ $\left.\left.+2 g n_{c}\right)\right]^{1 / 2}$ in terms of the bare polariton dispersion at linear regime. Within the parabolic approximation, this can be written as $\varepsilon_{k}=\hbar k^{2} / 2 m_{L P}$. As discussed in Ref. 16, the effective width $\Gamma$ is defined as $\Gamma=\alpha \beta \gamma /(1+\alpha \beta)$, in terms of the scaled pump intensity $\alpha=P / P_{\text {th }}-1$ above the threshold value $P_{\text {th }}$ and the $\beta$ coefficient defined in terms of gain rate $R\left(n_{R}\right)$ of the condensate from the excitonic reservoir as $\beta$ $=n_{R}^{0} R^{\prime}\left(n_{R}^{0}\right) / R\left(n_{R}^{0}\right)$. Well above the threshold $\alpha \gg 1$, the effective width $\Gamma$ recovers the empty-cavity polariton decay rate $\gamma$.

Note how the dispersion of elementary excitations significantly differs from the naive Hartree prediction $\omega=\omega_{0}$ $+2 g_{R} n_{R}+g n_{c}+\hbar k^{2} / 2 m$ (dashed line) and rather recovers in the high-momentum region the value $\omega=\omega_{0}+2 g_{R} n_{R}+2 g n_{c}$ $+\hbar k^{2} / 2 m$ which correctly includes a factor of 2 due to bosonic exchange (solid line). Provided that the effect of saturation ${ }^{24}$ on the polariton interactions is negligible, these simple arguments can be used to isolate the contribution of, respectively, the condensate-reservoir and the condensatecondensate interactions to the blue shift and possibly even evaluate the fraction of condensed excitons with respect to the total number of excitons in the microcavity.

\section{FOUR-WAVE MIXING SPECTROSCOPY SCHEME}

Even though a simple luminescence experiment should in principle be able to reveal both the positive and the negative ghost branches of the elementary excitation spectrum shown in Fig. 1, experimental observations have not been able to provide unambiguous evidence of the ghost branch yet. ${ }^{1,3,13}$ Most likely the very weak luminescence coming from the ghost branch is hidden by the much stronger emission from the condensate and the upper branch.

The physical process underlying our proposal is sketched in Fig. 1. Elementary excitations are created on top of the condensate by injecting extra polaritons with a probe laser beam at a finite in-plane wave vector $\mathbf{k}$ tuned at a frequency $\omega$. The response of the system is then observed via the coherent light emission at an opposite wave vector $\mathbf{k}$ at an energy $2 \omega_{c}-\omega$ : the existence of a coherent coupling between the symmetrically located frequencies $\omega$ and $2 \omega_{c}-\omega$ and wave vectors $\pm \mathbf{k}$ stems from the fact that the elementary excitations of the condensate consist of a coherent superposition of plane waves at $(\mathbf{k}, \omega)$ and $\left(-\mathbf{k}, 2 \omega_{c}-\omega\right){ }^{9,25}$ Equivalently, this same process can be interpreted in nonlinear optical terms as a stimulated parametric scattering where a pair of condensate polaritons is coherently scattered into one more probe polariton plus one FWM polariton of symmetric wave vector and frequency. Within the standard language of 
four-wave mixing, two of the three input beams are provided by the condensate itself and only the third comes from the incident laser.

\section{A. Transmittivity}

The same linearization procedure that was used in Ref. 16 to obtain the dispersion of the elementary excitations can be straightforwardly used to determine the response of the system to a (weak) probe laser of amplitude $E_{\mathrm{pr}}(\mathbf{k}, \omega)$. The simplest quantity to consider is the probe transmission.

In the usual approximation that the effective reservoir relaxation rate $\gamma_{R}$ is much larger than all other frequency scales in the problem, the reservoir dynamics can be eliminated and the amplitude of the transmitted field has the following form:

$$
E_{\mathrm{tr}}(\mathbf{k}, \omega)=\frac{\sqrt{T_{0}} \gamma}{2} \frac{g n_{c}+\varepsilon_{\mathbf{k}}+\omega+i \Gamma / 2}{\left[\omega-\omega_{+}(\mathbf{k})\right]\left[\omega-\omega_{-}(\mathbf{k})\right]} E_{\mathrm{pr}}(\mathbf{k}, \omega) .
$$

The frequencies $\omega_{ \pm}(\mathbf{k})$ that appear in the resonant denominators were defined in Eq. (1): the transmission is resonantly enhanced when the probe is on resonance with an elementary excitation of the polariton condensate, either on the positive energy branch or on the negative energy ghost branch. A complete density plot of the transmittivity $T=\left|E_{\mathrm{tr}} / E_{\mathrm{pr}}\right|^{2}$ in the $(\omega, k)$ plane is shown in Fig. 2(a). For the sake of simplicity, the transmission $T$ is normalized to the linear regime resonant transmittivity $T_{0}$ of the unloaded cavity.

At large momenta $k$, the intensity of the ghost mode is much weaker than the one of the upper, normal mode and quickly tends to zero. Already for $k \xi=1$, the ghost resonance is extremely weak and almost invisible in the cut shown as a dotted line in Fig. 2(c). On the other hand, the normal resonance tends to the unloaded cavity peak of height $T / T_{0}=1$ and width $\gamma$. For decreasing values of $k$, the intensities of the two resonances get closer and eventually stick at the singular point $k \xi=0.5$ of the dispersion [Fig. 2(e)].

In the low- $k$ diffusive region where the two branches are degenerate $\operatorname{Re}\left[\omega_{ \pm}\right]=0$, the transmission intensity significantly exceeds the peak transmittivity $T_{0}$ of the unloaded cavity [solid line in Fig. 2(c)]: this gain feature is due to the condensate that is able to amplify the probe beam. A similar phenomenology was found and discussed in Ref. 15 for the case of an optical parametric oscillator.

While the appearance of the ghost branch in the transmission spectra of Fig. 2 is a signature of the presence of the condensate, the transmittivity on the normal branch can exceed the linear regime value $T_{0}$ even for pump intensities well below the condensation threshold. Even if not sufficient to overcome losses, some gain is in fact present also in this case, ${ }^{17}$ as witnessed by the polariton linewidth decreasing below the unloaded cavity value $\gamma$.

\section{B. FWM signal}

The same method can be used to obtain an expression for the amplitude of the four-wave mixing signal
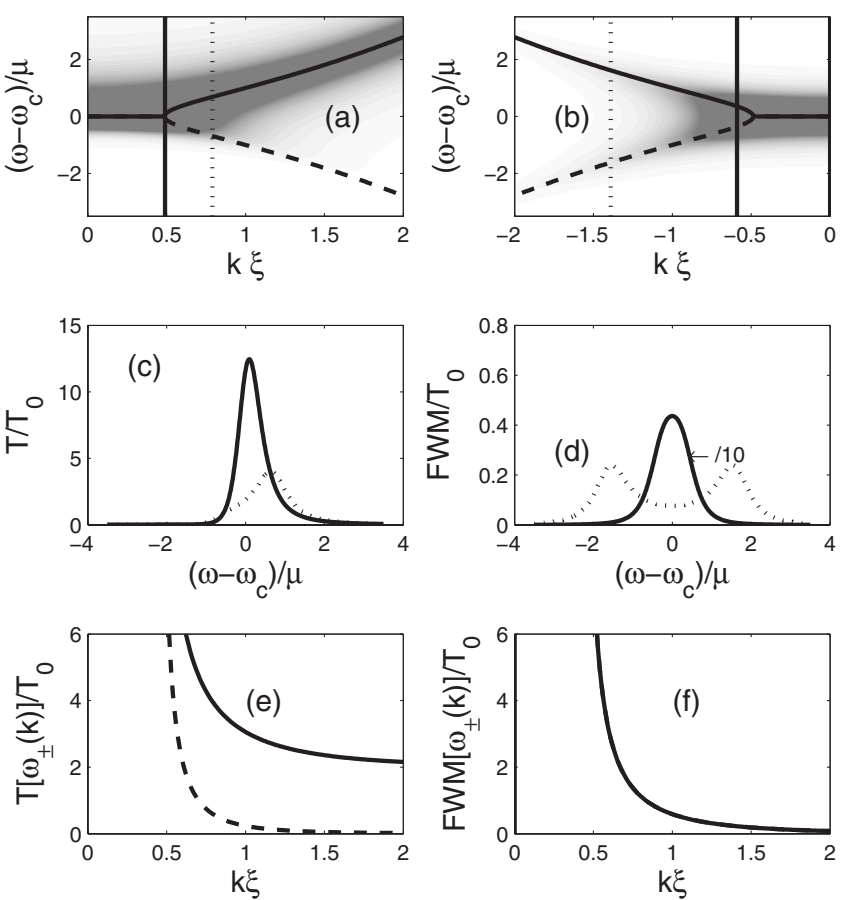

FIG. 2. (a) Transmittivity and (b) FWM spectra of a nonequilibrium condensate excited with a probe laser at wave vector $k>0$ and frequency $\omega$. The full and dashed lines indicate the positive and negative Bogoliubov branches from Eq. (1). Cuts of the transmission and FWM spectra at given $k$ values are shown in panels (c) and (d); the corresponding $k$ values are indicated by the vertical lines in panels (a) and (b). The peak value of the transmittivity and the FWM signal on, respectively, the normal and ghost Bogoliubov branches are shown in (e) and (f) as solid and dashed lines. In (f) the two quantities coincide. All spectra are normalized to the empty-cavity, linear regime transmittivity $T_{0}$. System parameters: $\Gamma=\gamma=g n_{c}$.

$$
\begin{aligned}
E_{\mathrm{FWM}}(\mathbf{k}, \omega)= & -\frac{\sqrt{T_{0}} \gamma}{2} \frac{g n_{c}+i \Gamma / 2}{\left[\omega-\omega_{+}(\mathbf{k})\right]\left[\omega-\omega_{-}(\mathbf{k})\right]} \\
& \times E_{\mathrm{pr}}^{*}\left(-\mathbf{k}, 2 \omega_{c}-\omega\right) .
\end{aligned}
$$

The response function $S_{\mathrm{FWM}}=\left|E_{\mathrm{FWM}} / E_{\mathrm{pr}}\right|^{2}$ is plotted in the upper-left panel of Fig. 2. Thanks to the symmetry under the exchange $\omega \rightarrow-\omega$, the heights of the two peaks corresponding to the normal and the ghost branches are equal.

This is a crucial advantage of the FWM technique as compared to the transmission spectroscopy discussed in Sec. III A or the resonant Rayleigh scattering discussed in Ref. 18. Different from this latter scheme, FWM does not rely on the presence of a disorder potential and therefore is not affected by specklelike modulations of the detected signal. As it happened for the probe transmission, also the FWM signal results as strongly enhanced in the diffusive region and may eventually become stronger than the probe itself. On the other hand, it decreases quite quickly for larger values of $k$.

Note that both direct polariton-polariton collisions and nonlinear gain saturation effects contribute to the FWM signal via, respectively, the terms $g n_{c}$ and $i \Gamma / 2$ in the numerator of Eq. (3): some FWM signal is therefore expected to appear 
even for negligible polariton-polariton collisions but is in this case peaked very close to the free polariton dispersion. On the other hand, the presence of a FWM signal appears to be conclusive evidence of the presence of a coherent condensate: the same calculation below threshold would in fact give a vanishing coherent FWM amplitude in the weak probe limit. Gain saturation is in fact not active in this regime and FWM processes involving the exciton reservoir only provide an incoherent background without any specific resonant feature.

\section{Reflectivity}

As many microcavity samples are grown on an absorbing substrate, transmission measurements are not always possible. While FWM expression (3) is the same in both reflection and transmission geometries, the interference between direct reflections of light on the external mirror and the secondary emission from the cavity makes the following expression of the reflection amplitude a bit more complicate than transmission amplitude (2):

$$
E_{r}(\mathbf{k}, \omega)=\left(1-i \frac{\gamma_{\mathrm{ph}}}{2} \frac{g n_{c}+\varepsilon_{\mathbf{k}}+\omega+i \Gamma / 2}{\left[\omega-\omega_{+}(\mathbf{k})\right]\left[\omega-\omega_{-}(\mathbf{k})\right]}\right) E_{\mathrm{pr}}(\mathbf{k}, \omega) .
$$

Note that the linewidth $\gamma_{\mathrm{ph}}$ that appears in the numerator of Eq. (4) is the linewidth of the cavity-photon mode in the absence of any excitonic resonance. This quantity is to be distinguished from the linewidth $\gamma$ of the polariton branch that appeared in the previous formulas. In terms of the Hopfield coefficients $U_{\mathrm{ph}, x}^{\mathrm{LP}}$ that quantify the cavity-photonic and the excitonic contents of the lower polariton branch, the polariton $\gamma$, exciton, and cavity-photon $\gamma_{x, \mathrm{ph}}$ linewidths are related by ${ }^{24}$

$$
\gamma=\gamma_{x}\left|U_{x}^{\mathrm{LP}}\right|^{2}+\gamma_{\mathrm{ph}}\left|U_{c}^{\mathrm{LP}}\right|^{2} .
$$

As it involves subtle interference effects, the reflection spectrum turns out to be sensitive to the ratio of the linewidth of excitations in the empty and pumped cavity $\gamma / \Gamma \geq 1$, as well as to the ratio between the cavity-photon and the polariton linewidths $\gamma_{\mathrm{ph}} / \gamma$. Here we focus our attention on the experimentally most relevant case where $\gamma_{\mathrm{ph}}=\gamma_{x}=\gamma$. Examples of spectra are shown in Fig. 3 for different values of the pumping intensity. Panels (a) and (c) refer to a case well above threshold for which $\Gamma / \gamma \simeq 1$, while panels (b) and (d) are made for a lower (but still above threshold) value of the pump intensity at which $\Gamma / \gamma=0.4$.

In panel (a), a minimum in the reflectivity is visible on resonance with the positive Bogoliubov branch, while the reflected intensity on resonance with the negative branch exceeds the incident intensity. As for the transmission and the FWM signal, the amplification effect is the strongest in the diffusive region at low $k$ [panel (c)]. At high values of $k$, the dip corresponding to the normal branch remains fully visible, while the peak corresponding to the ghost branch disappears. This once again confirms that the FWM mixing scheme is the most suited tool for the detection of the ghost branch.

The same quantities are plotted in panels (b), (d), and (f) for a smaller value of $\Gamma / \gamma=0.4$, i.e., for a pump intensity
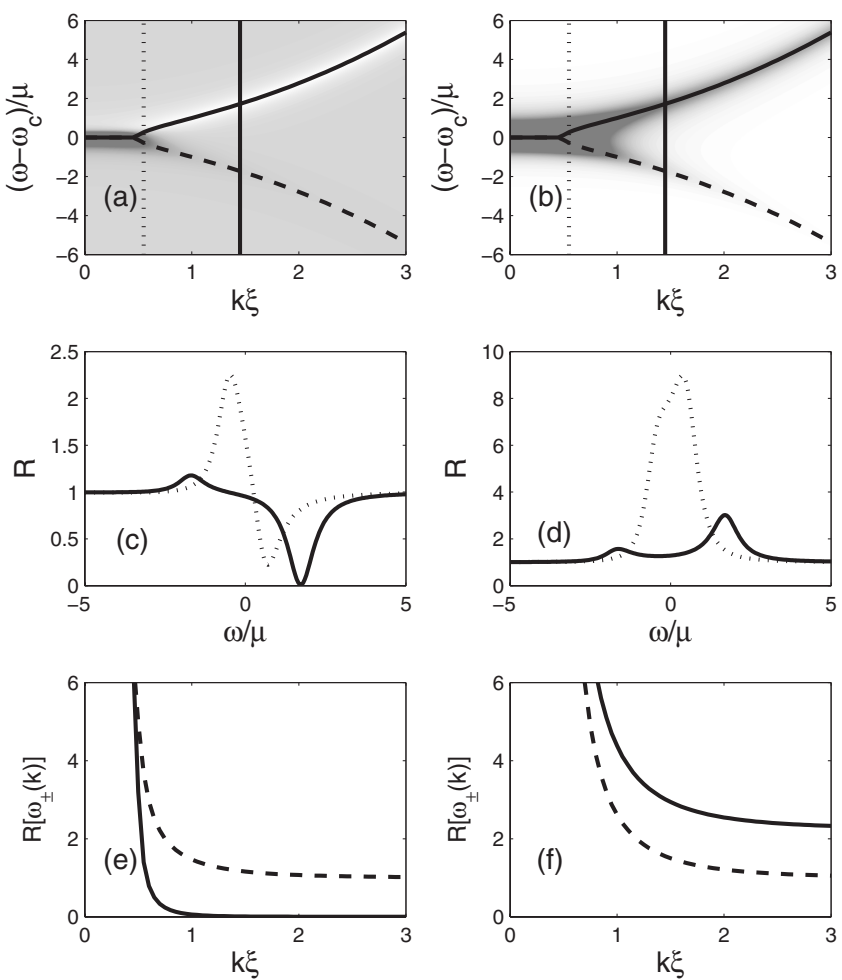

FIG. 3. Panels (a) and (b): $(k, \omega)$ plot of the reflectivity for (a) $\gamma=\Gamma=g n_{c}$ and (b) $\Gamma=0.4 \gamma=g n_{c}$. (c) and (d) show reflection spectra at fixed values of $k$ as indicated by the vertical lines in panels (a) and (b). The peak values of the reflectivity on the normal and ghost Bogoliubov branches are, respectively, shown in (e) and (f) as solid and dashed lines. Parameters: $\gamma=\gamma_{\mathrm{ph}}=\gamma_{x}$.

closer to the threshold. As a consequence of the narrower resonance line, the reflected beam is now more intense than the incident one on both the normal and the ghost branches. The amplification in the low- $k$ diffusive region is also stronger than in the case $P \gg P_{\text {th }}$ considered before.

\section{PROBING THE ELEMENTARY EXCITATION SPECTRUM IN FINITE-SIZE AND/OR DISORDERED CONDENSATES}

Even in the absence of trapping potentials, the spatial extension of polariton condensates is generally limited by the size of the laser spot that is used to pump the microcavity. ${ }^{22}$ Typically, state-of-the-art polariton condensates have a typical size on the order of tens of microns and may show a considerable inhomogeneous broadening of their spectral features as a consequence of the inhomogeneous density profile. Although this effect can be reduced by choosing top-hat pump beams, the interpretation of FWM experiments is the clearest if one focuses the probe spot onto a small region at the center of the condensate where the density is almost flat and one spatially selects the emission from the same central region.

The result of numerical calculations based on the full polariton Gross-Pitaevskii equation ${ }^{16}$ is summarized in Fig. 4. The case of a probe beam of diameter $1 \mu \mathrm{m}$ much smaller than the full condensate size and tuned at a frequency 


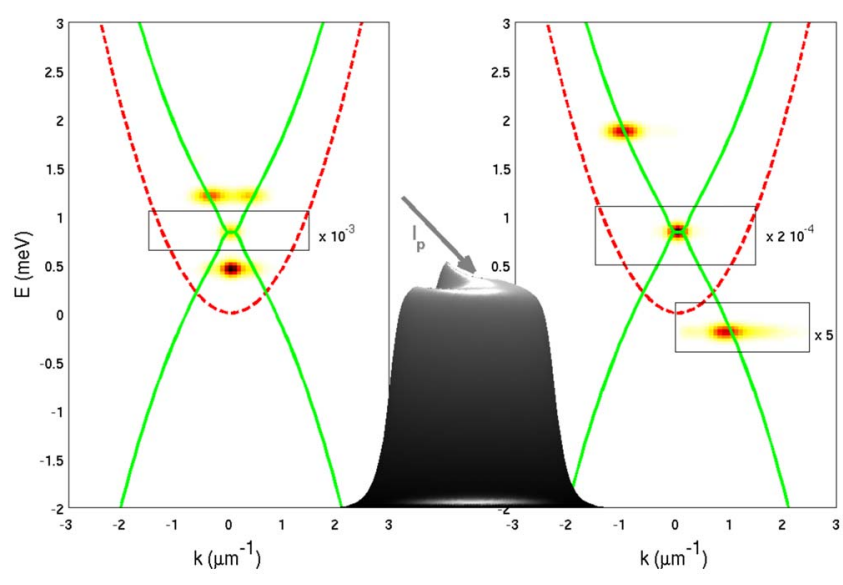

FIG. 4. (Color online) Numerical results for the $(k, \omega)$ emission pattern from a probed polariton condensate in a transmission geometry. The condensate frequency is $\omega_{c}=0.83 \mathrm{meV}$ and probe laser has a $1 \mu \mathrm{m}$ diameter. The central wave vector and the frequency of the probe are $k_{p}=0.6 \mu \mathrm{m}^{-1}$ and $\omega_{p}=0.3 \mathrm{meV}$ (left panel) and $k_{p}$ $=1.5 \mu \mathrm{m}^{-1}$ and $\omega_{p}=-0.2 \mathrm{meV}$ (right panel). The full and dashed lines show the analytical prediction, the excitation spectrum, and the linear regime polariton dispersion. The central surface plot shows the real-space polariton density profile corresponding to the right-hand panel. In all panels, the condensate is excited with a top-hat laser with a $30 \mu \mathrm{m}$ diameter and only the emission originating from a central region with a $20 \mu \mathrm{m}$ diameter is recorded in the left and right panels. The finite frequency spread of the emission lines originates from the finite time window of about 12 ps from which the spectrum was extracted.

slightly below the condensate frequency $\omega_{c}$ is considered.

The case of a quite high probe wave vector $k_{p}$ $=1.5 \mu \mathrm{m}^{-1}$ tuned close to resonance with the ghost branch is shown in the right panel of Fig. 4. As the $k$ distribution is narrow as compared to the central wave vector $k_{p}$, the response closely resembles the one of the spatially homogeneous case discussed in Sec. III. A single FWM response peak appears around $k_{p}$ and with a $k$-space linewidth narrower than the one of the incident probe: only those wave vector components that are on resonance with the ghost branch effectively contribute to the FWM signal.

A remarkable feature of the ghost branch is visible in the real-space density profile of the probed condensate shown in the central panel of Fig. 4: as the group velocity of the ghost branch is opposite to the wave vector $\mathbf{k}$, the perturbation due to the probe laser (with a central wave vector $k_{p}>0$ pointing to the right) propagates through the condensate in the leftwards direction and concentrates on the left-hand side of the probe spot.

The case of a probe laser with a smaller wave vector and a frequency closer to but still below the condensate frequency is shown in the left panel. This choice dramatically modifies the qualitative shape of the response. In particular, note how the maximum of the response at the probe frequency lies between the two excitation branches of the homogeneous system, a spectral region where collective modes are strongly affected by the finite size of the system and possibly by the nontrivial spectral shape of the condensate. $^{22,26}$ Although information on the excitation modes of

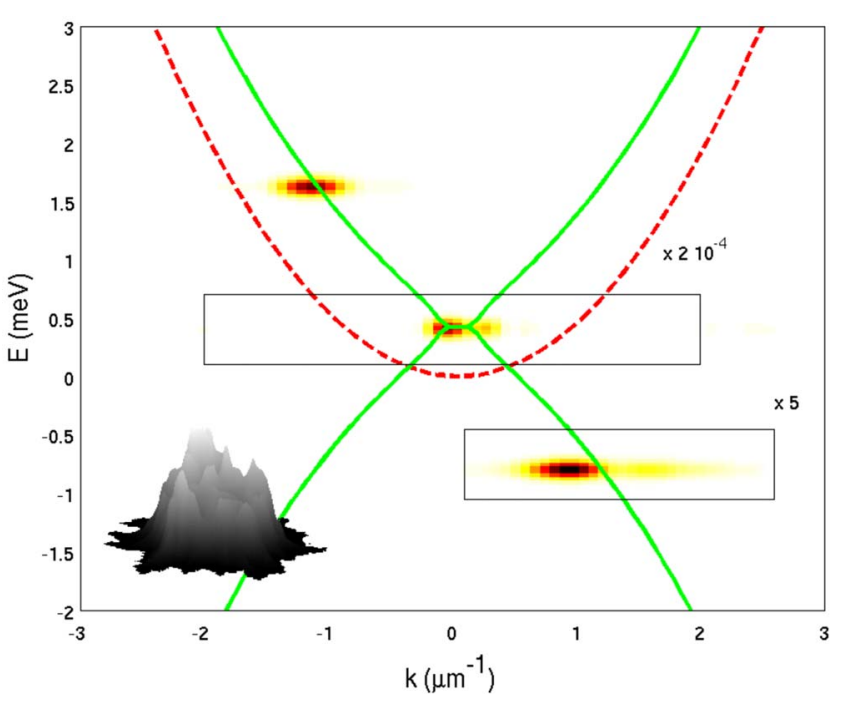

FIG. 5. (Color online) Emission pattern for the same configuration as in the right-hand panel of Fig. 4 but in the presence of a disorder potential acting on the polaritons. The height of the disorder potential is in the $1 \mathrm{meV}$ range. Inset: density profile of the disordered condensate.

the condensate is hardly obtained in this configuration, the very presence of a FWM signal is still a direct proof of the presence of a coherent condensate.

The situation is a bit more complex in the presence of disorder. As any experimental sample is inevitably far from being perfectly homogeneous, it is important to briefly summarize the main features that we observe in our numerical simulations when a disorder potential acting on polaritons is included. A complete study is postponed to further work.

The case of a weak disorder is illustrated in Fig. 5: the strength of the disorder potential is chosen to be strong enough to substantially modulate the polariton density (see inset) but weak enough not to split the condensate into several frequency components. ${ }^{27}$ In this case, the FWM signal remains clearly visible: the wave vector distribution is strongly broadened by the disorder, but the main features that were observed in the right panel of Fig. 4 are still apparent.

The situation is dramatically different whenever multiple condensates are present with a substantial spatial overlap. In this case, the main effect of the probe beam is to redistribute the intensity among the different condensate frequencies and no clear FWM signal is easily identified among the many spectral lines forming the emission spectrum.

\section{CONCLUSIONS}

We have proposed and analyzed a four-wave mixing spectroscopy scheme to probe the excitation spectrum of a nonequilibrium polariton condensate. Clear evidence of the negative frequency ghost branch is predicted to appear in the FWM spectra. The presence of this feature depends crucially on the coherent nature of the condensate and can therefore be used as an additional probe of the condensate coherence. Mapping out the FWM resonance as a function of frequency 
and wave vector gives precise access to the elementary excitation spectrum. We have numerically demonstrated that a spatially localized probe can be used to overcome inhomogeneous broadening effects by restricting the measurement to the central region of the condensate where the density is almost flat.

\section{ACKNOWLEDGMENTS}

We wish to thank V. Savona, D. Sarchi, M. Richard, K. Lagoudakis, A. Baas, and B. Pietka for continuous stimulating discussions on polariton condensates. I.C. acknowledges financial support from the Italian MIUR, the EuroQUAMFerMix program, and the French IFRAF.
${ }^{1}$ J. Kasprzak et al., Nature (London) 443, 409 (2006).

${ }^{2}$ H. Deng, D. Press, S. Gotzinger, G. S. Solomon, R. Hey, K. H. Ploog, and Y. Yamamoto, Phys. Rev. Lett. 97, 146402 (2006).

${ }^{3}$ R. Balili, V. Hartwell, D. Snoke, L. Pfeiffer, and K. West, Science 316, 1007 (2007)

${ }^{4}$ S. Christopoulos, G. Baldassarri Höger von Högersthal, A. J. D. Grundy, P. G. Lagoudakis, A. V. Kavokin, J. J. Baumberg, G. Christmann, R. Butté, E. Feltin, J.-F. Carlin, and N. Grandjean, Phys. Rev. Lett. 98, 126405 (2007).

${ }^{5}$ D. Bajoni, P. Senellart, E. Wertz, I. Sagnes, A. Miard, A. Lemaitre, and J. Bloch, Phys. Rev. Lett. 100, 047401 (2008).

${ }^{6}$ S. O. Demokritov, V. E. Demidov, O. Dzyapko, G. A. Melkov, A. A. Serga, B. Hillebrands, and A. N. Slavin, Nature (London) 443, 430 (2006).

${ }^{7}$ Phase Transitions and Critical Phenomena, Statistical Mechanics of Driven Diffusive Systems Vol. 17, edited by C. Domb and J. L. Lebowitz (Academic, New York, 1989).

${ }^{8}$ A. L. Leggett, Rev. Mod. Phys. 71, S318 (1999).

${ }^{9}$ L. P. Pitaevskii and S. Stringari, Bose-Einstein Condensation (Clarendon, Oxford, 2003).

${ }^{10}$ P. G. Savvidis, C. Ciuti, J. J. Baumberg, D. M. Whittaker, M. S. Skolnick, and J. S. Roberts, Phys. Rev. B 64, 075311 (2001); A. I. Tartakovskii, D. N. Krizhanovskii, D. A. Kurysh, V. D. Kulakovskii, M. S. Skolnick, and J. S. Roberts, ibid. 65, 081308(R) (2002).

${ }^{11}$ J. J. Baumberg and P. G. Lagoudakis, Phys. Status Solidi B 242, 2210 (2005)

${ }^{12}$ D. Ballarini, D. Sanvitto, A. Amo, L. Viña, M. Wouters, I. Carusotto, A. Lemaître, and J. Bloch, arXiv:0807.3224 (unpublished).

${ }^{13}$ S. Utsunomiya, L. Tian, G. Roumpos, C. W. Lai, N. Kumada, T. Fujisawa, M. Kuwata-Gonokami, A. Löffler, S. Höfling, A. Forchel, and Y. Yamamoto, Nat. Phys. 4, 700 (2008).

${ }^{14}$ M. H. Szymańska, J. Keeling, and P. B. Littlewood, Phys. Rev.
Lett. 96, 230602 (2006).

${ }^{15}$ M. Wouters and I. Carusotto, Phys. Rev. A 76, 043807 (2007).

${ }^{16}$ M. Wouters and I. Carusotto, Phys. Rev. Lett. 99, 140402 (2007).

${ }^{17}$ P. R. Eastham and P. B. Littlewood, Phys. Rev. B 64, 235101 (2001).

${ }^{18}$ F. M. Marchetti, J. Keeling, M. H. Szymańska, and P. B. Littlewood, Phys. Rev. B 76, 115326 (2007).

${ }^{19}$ D. Sarchi and V. Savona, Phys. Status Solidi C 5, 2420 (2008).

${ }^{20}$ A. Huynh, J. Tignon, O. Larsson, Ph. Roussignol, C. Delalande, R. André, R. Romestain, and Le Si Dang, Phys. Rev. Lett. 90, 106401 (2003).

${ }^{21}$ M. Richard, J. Kasprzak, R. Romestain, R. André, and L. S. Dang, Phys. Rev. Lett. 94, 187401 (2005).

${ }^{22}$ M. Wouters, I. Carusotto, and C. Ciuti, Phys. Rev. B 77, 115340 (2008).

${ }^{23}$ K. G. Lagoudakis, M. Wouters, M. Richard, A. Baas, I. Carusotto, R. André, Le Si Dang, and B. Deveaud-Plédran, Nat. Phys. 4, 706 (2008).

${ }^{24}$ C. Ciuti, P. Schwendimann, and A. Quattropani, Semicond. Sci. Technol. 18, S279 (2003).

${ }^{25}$ Y. Castin, in Coherent Atomic Matter Waves: Lecture Notes of Les Houches Summer School, edited by R. Kaiser, C. Westbrook, and F. David (EDP Sciences and Springer-Verlag, Berlin, 2001), pp. 1-136, and references therein.

${ }^{26}$ This interpretation is supported by the numerical observation that the response is again peaked on the Bogoliubov excitation branch if a larger condensate is considered.

${ }^{27}$ A. Baas, K. G. Lagoudakis, M. Richard, R. André, Le Si Dang, and B. Deveaud-Plédran, Phys. Rev. Lett. 100, 170401 (2008); A. P. D. Love, D. N. Krizhanovskii, D. M. Whittaker, R. Bouchekioua, D. Sanvitto, S. Al. Rizeiqi, R. Bradley, M. S. Skolnick, P. R. Eastham, R. André, and Le Si Dang, ibid. 101, 067404 (2008). 